This item was submitted to Loughborough's Research Repository by the author.

Items in Figshare are protected by copyright, with all rights reserved, unless otherwise indicated.

\title{
An applicable approach for extracting human heart rate and oxygen saturation during physical movements using a multi-wavelength illumination optoelectronic sensor system
}

\section{PLEASE CITE THE PUBLISHED VERSION}

https://doi.org/10.1117/12.2287854

\section{PUBLISHER}

(C) 2018 Society of Photo-Optical Instrumentation Engineers (SPIE).

\section{VERSION}

VoR (Version of Record)

\section{LICENCE}

CC BY-NC-ND 4.0

\section{REPOSITORY RECORD}

Alharbi, Samah, Sijung Hu, David J. Mulvaney, and Panagiotis Blanos. 2019. "An Applicable Approach for Extracting Human Heart Rate and Oxygen Saturation During Physical Movements Using a Multi-wavelength Illumination Optoelectronic Sensor System". figshare. https://hdl.handle.net/2134/33378. 


\section{An applicable approach for extracting human heart rate and oxygen saturation during physical movements using a multi-wavelength illumination optoelectronic sensor system}

Samah Alharbi, Sijung Hu, David Mulvaney, Panagiotis Blanos

Samah Alharbi, Sijung Hu, David Mulvaney, Panagiotis Blanos, "An applicable approach for extracting human heart rate and oxygen saturation during physical movements using a multi-wavelength illumination optoelectronic sensor system," Proc. SPIE 10486, Design and Quality for Biomedical Technologies XI, 104860S (19 February 2018); doi: 10.1117/12.2287854

SPIE. Event: SPIE BiOS, 2018, San Francisco, California, United States 


\title{
An applicable approach for extracting human heart rate and oxygen saturation during physical movements using a multi-wavelength illumination optoelectronic sensor system
}

\author{
Samah Alharbi ${ }^{1}$, Sijung $\mathrm{Hu}^{1 *}$, David Mulvaney ${ }^{1}$ and Panagiotis Blanos ${ }^{1}$ \\ ${ }^{1}$ Wolfson School of Mechanical, Electrical and Manufacturing Engineering, Loughborough \\ University, Ashby Road, Loughborough, Leicestershire LE11 3TU UK
}

\begin{abstract}
The ability to gather physiological parameters such as heart rate (HR) and oxygen saturation $(\mathrm{SpO} 2 \%)$ during physical movement allows to continuously monitor personal health status without disrupt their normal daily activities. Photoplethysmography (PPG) based pulse oximetry and similar principle devices are unable to extract the HR and $\mathrm{SpO} 2 \%$ reliably during physical movement due to interference in the signals that arise from motion artefacts (MAs). In this research, a flexible reflectance multi-wavelength optoelectronic patch sensor (OEPS) has been developed to overcome the susceptibility of conventional pulse oximetry readings to MAs. The OEPS incorporates light embittered diodes as illumination sources with four different wavelengths, e.g. green, orange, red, and infrared unlike the conventional pulse oximetry devices that normally measure the skin absorption of only two wavelengths (red and infrared). The additional green and orange wavelengths were found to be distinguish to the absorption of deoxyhemoglobin $(\mathrm{RHb})$ and oxyhemoglobin $(\mathrm{HbO} 2)$. The reliability of extracting physiological parameters from the green and orange wavelengths is due to absorbed near to the surface of the skin, thereby shortening the optical path and so effectively reducing the influence of physical movements. To compensate of MAs, a three-axis accelerometer was used as a reference with help of adaptive filter to reduce MAs. The experiments were performed using 15 healthy subjects aged 20 to 30 . The primary results show that there are no significant difference of heart rate and oxygen saturation measurements between commercial devices and OEPS Green $(\mathrm{r}=0.992)$, Orange $(\mathrm{r}=0.984), \operatorname{Red}(\mathrm{r}=0.952)$ and $\mathrm{IR}(\mathrm{r}=0.97)$ and $\mathrm{SpO} 2 \%(\mathrm{r}=0.982, \mathrm{p}=0.894)$.
\end{abstract}

Keywords: Heart rate monitoring, Oxygen saturation monitoring, Optoelectronic patch sensor, deoxyhemoglobin, oxyhemoglobin.

\section{INTRODUCTION}

With the goal of improving quality of life, many researchers have attempted to improve the performance of systems for monitoring physiological information. One of the methods commonly used for early detection and continuous monitoring is Photoplethysmography (PPG), a non-invasive optical technique able to measure volume changes in blood vessels and so estimate vital health signs, such as heart rate (HR), heart rate variability, respiration rate, blood pressure and oxygen saturation $\left(\mathrm{SpO}_{2} \%\right)^{[1]}$. There are two main types of operation used in PPG sensors, namely transmission mode and reflectance mode. In transmission mode, a photo detector (PD) is placed to detect light after it has travelling through the subject's body part (such as a finger or earlobe). In reflectance mode, the PD is located in the same side of the body part as the source and captures back-scattered light from the skin and tissue. Transmission mode is widely used in hospital and clinical settings, such as in pulse oximetry, whereas the reflectance approach is mainly used in wearable sensors, such as smartwatches.

\footnotetext{
*S.Hu@lboro.ac.uk; phone +44 1509227059
}

Design and Quality for Biomedical Technologies XI, edited by Ramesh Raghavachari, Rongguang Liang, Proc. of SPIE Vol. 10486, 104860S · C 2018 SPIE · CCC code: 1605-7422/18/\$18 · doi: 10.1117/12.2287854 
In the work described in this paper, reflectance mode is used. The raw reflected PPG signal is often considered to be composed of two separate components, namely a direct current (DC) and an alternating current (AC) component ${ }^{[2]}$. The DC component arises from non-pulsatile tissue such as bones and veins that maintain a constant absorption characteristic during measurement. The $\mathrm{AC}$ component is the variation in the intensity of reflected light that results from the changing absorption characteristics as the blood pulses through tissue. The amplitude of the AC component is typically $1 \%$ to $2 \%$ of that of the DC component ${ }^{[3]}$, making its extraction more susceptible to the presence of measurement and electrical noise.

To date, a number of wearable sensors capable of detecting physiological parameters have been developed commercially, and these include as HRM-3200 (H3, Korea) ${ }^{[4]}$, Honour S1 (Huawei, China) ${ }^{[5]}$, MZ-3Belt (Myzone, UK ${ }^{[6]}$, Nonin WristOx 3100[7], AMON (Advanced Medical Monitor) physiological monitor ${ }^{[8]}$ LifeShirt ${ }^{[9]}$, Smart Shirt ${ }^{[10]}$, SenseWear ${ }^{[11]}$ and Smart Vest ${ }^{[12]}$.

Most PPG probe sensors currently in use in hospitals and clinics are designed to operate in transmission mode, which is restrictive in terms of the number of options available for the measurement site (typically the only options are the finger, the earlobe and the toe), as well as limiting the freedom of movement available to patients ${ }^{[2]}$ (for example, fingertip sensors, such as those use for pulse oximetry, interfere with daily activities and it can be difficult to measure vital signs during physical movement). By using a reflectance multi-wavelength optoelectronic sensor in this work, the number of suitable sites available on the body increases dramatically. Further, it has been reported that the reflectance mode PPG sensor probe, MaxFast (Nellcor ${ }^{\mathrm{TM}}$, USA), has been used clinically to provide non-invasive continuous monitoring, albeit giving occasional erroneous readings ${ }^{[2]}$. We propose that further research into reflectance mode is needed with the aim of determining whether this approach will improve comfort and convenience for patients when taking PPG measurements.

Current pulse oximetry and PPG sensors mainly use two separate wavelengths, namely red and infra-Red (IR). However, these two wavelengths have been reported to produce signals of poor reliability during physical movement ${ }^{[13]}$. It would be appropriate to consider green as an alternative light color as it has been reported to provide signals less prone to be affected by movements ${ }^{[2]}$. In addition, the measurement of certain physiological parameters, such as $\mathrm{SpO}_{2} \%$, needs signals obtained from incident light of at least two different wavelengths and products such as smartwatches mostly use only one wavelength and consequently are restricted to determining only HR. In this study, a multi-wavelength illumination optoelectronic sensor is used and will allow the measurement of both $\mathrm{HR}$ and $\mathrm{SpO}_{2} \%$.

This paper introduces an reflective-mode optoelectronic patch sensor (OEPS) that provides illumination at of four wavelengths, namely green $(525 \mathrm{~nm})$, orange $(590 \mathrm{~nm})$, red $(650 \mathrm{~nm})$ and IR $(870 \mathrm{~nm})$ and has been designed to allow the measurement of $\mathrm{HR}$ and $\mathrm{SpO}_{2} \%$. By using a multi-wavelength reflective-mode PPG, the work described in this study is able to operate under a number of different scenarios, including during physical movement ${ }^{[14]}$, with the sensor located at a broader range of measurement sites ${ }^{[15]}$, applied to subjects with a range of skin pigments ${ }^{[16]}$ and with the potential for application in childcare.

\section{OXYGEN SATURATION MEASUREMENT}

Pulse oximeters are non-invasive devices designed to measure $\mathrm{SpO}_{2} \%$. Haemoglobin is the oxygen-transport metalloprotein in the red blood cells ${ }^{[17]}$ and as the light absorption of oxy-haemoglobin $\left(\mathrm{HbO}_{2}\right)$ is different from that of deoxyhaemoglobin $(\mathrm{Hb})$ at different wavelengths of the spectral window, then, from a ratio of the absorptions at two different wavelengths, $\mathrm{SpO}_{2} \%$ can be determined. Figure 2.1 shows the absorption of haemoglobin under red and IR illumination, with $\mathrm{Hb}$ absorbing relatively more red light than $\mathrm{HbO}_{2}$ and $\mathrm{Hb}$ relatively less IR light than $\mathrm{HbO}_{2}$. 


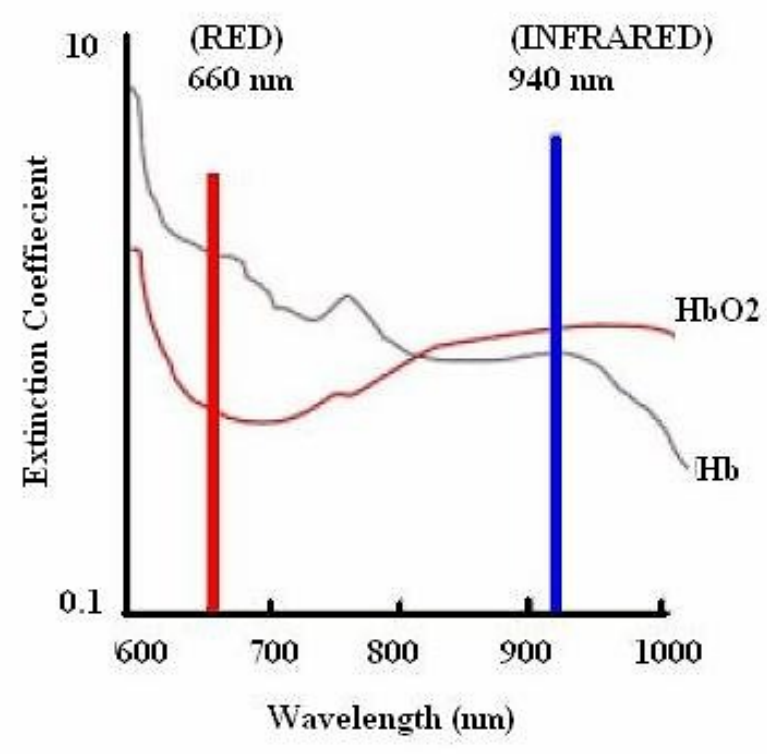

Figure 2.1 Absorption spectra of $\mathrm{HbO}_{2}$ and $\mathrm{Hb}^{[18]}$

As tissues in the body generally contain water, they generally absorb light more strongly in the longer wavelength IR region ${ }^{[3]}$, whereas melanin is known to absorb more light at shorter wavelengths ${ }^{[2]}$. Red blood cells are reported to be have greater light absorption at red and near IR wavelengths ${ }^{[19]}$ and this can be used to facilitate the measurement of blood volume change. As a consequence, red and IR wavelengths are commonly used as light sources in pulse oximetry ${ }^{[20]}$. There are several studies that have used red light $(650-750 \mathrm{~nm})^{[21,22]}$ and IR light $(850-1000 \mathrm{~nm})^{[23]}$ to measure HR and $\mathrm{SpO}_{2} \%$ using PPG. However, PPG signals are easily affected by sources of noise (human tissue deformation, ambient light interference and electromagnetic signals), making good estimates of physiological parameters difficult to obtain ${ }^{[24]}$. In this paper, green $(525 \mathrm{~nm})$ and orange $(590 \mathrm{~nm})$ wavelengths have been investigated as alternative light sources in the extraction of $\mathrm{SpO}_{2} \%$ values. Green and orange wavelengths are absorbed nearer to the outer layer of skin when compared to incident red and IR light. Consequently, although green and orange illumination penetrate the skin to a shallower depth and so do not capture blood pulse signals as effectively as red or IR light, their signal-to-noise ratio is improved as they suffer from less attenuation that results from their comparatively shorter path length ${ }^{[25]}$. Figure 2.2 shows the skin penetration depths of light signals of wavelengths from 400 to $1000 \mathrm{~nm}$.

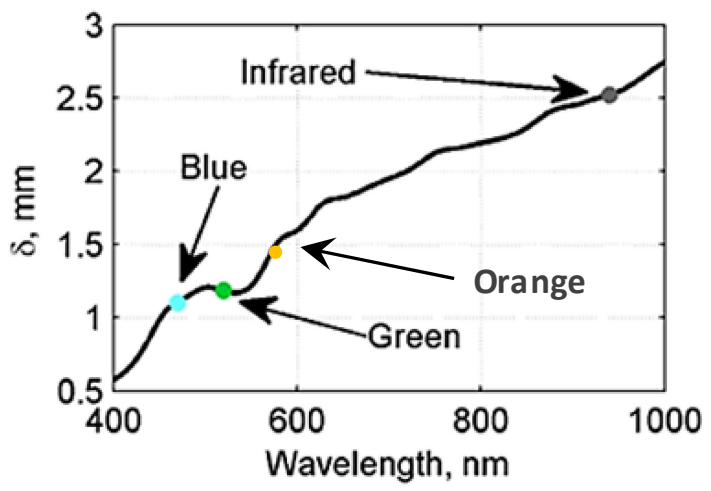

Figure 2.2 Optical penetration depth $\delta$ shown for a range of light sources of different wavelengths ${ }^{[24]}$ 
Figure 2.3 shows that there is a sufficiently significant difference in the absorption of $\mathrm{Hb}$ and $\mathrm{HbO}_{2}$ at both the green and orange wavelengths to make them suitable wavelengths for extracting $\mathrm{SpO}_{2} \%$ measurements.

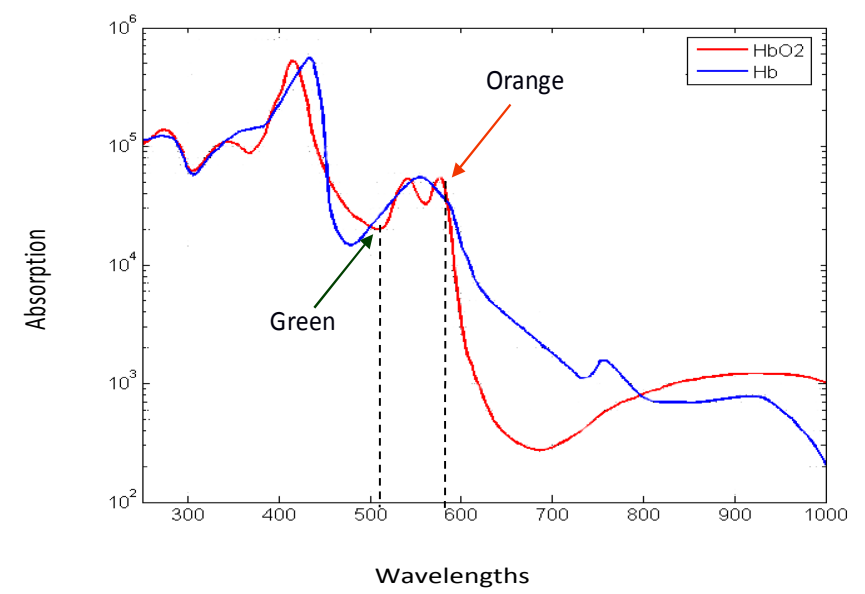

Figure 2.3 Absorption of $\mathrm{Hb}$ and $\mathrm{HbO}_{2}$ at green and orange wavelengths

\section{METHODOLOGY}

This section describes the approaches taken in the current work to the measurement of $\mathrm{HR}, \mathrm{SpO} 2 \%$ as well as providing a description of the sensing and processing systems.

\subsection{Physical Exercise Protocol}

Fifteen (15) healthy subjects (aged $25 \pm 5$ years), of height $178.9 \mathrm{~cm}( \pm 4.2 \mathrm{~cm})$ and body mass $70.9 \mathrm{~kg}( \pm 7.9 \mathrm{~kg})$ volunteered to take part in the study which was approved by the Loughborough University Ethical Advisory Committee. The protocol was designed to investigate the performance of the multi-wavelength OEPS sensor in the extraction of HR and $\mathrm{SpO}_{2} \%$ under different types of exercises and activities such as sitting, cycling walking and running as shown in Figure 3.1.1. The subjects were required to abstain from drinking alcohol, coffee or other substances that may affect their performance in the 24 hours leading up to the taking of the measurements. Subjects' data were recorded using the patch sensor placed in left the palm of the hand. In order to collect benchmark data, a number of commercial devices were worn either on the wrist or chest, and these were HRM-3200 (H3, Korea), Honour S1 (Huawei, China), pulse oximetry (TempIR ${ }^{\mathrm{TM}}$, China), MZ-3Belt (Myzone, UK) and H3+ digital holter ECG recorder (Mortara, U.S.). The data recording from these sensors and OEPS were processed and analysed using bespoke Matlab programs. The subjects were first asked to remain in a seated, upright position for one minute while the sensors were attached before raw data were captured over a period of three minutes. Time windows of $15 \mathrm{~s}$ duration were used during processing and for the calculation of $\mathrm{HR}$ and $\mathrm{SpO}_{2} \%$. Figure 3.1.2 shows the typical signals obtained from OEPS. 


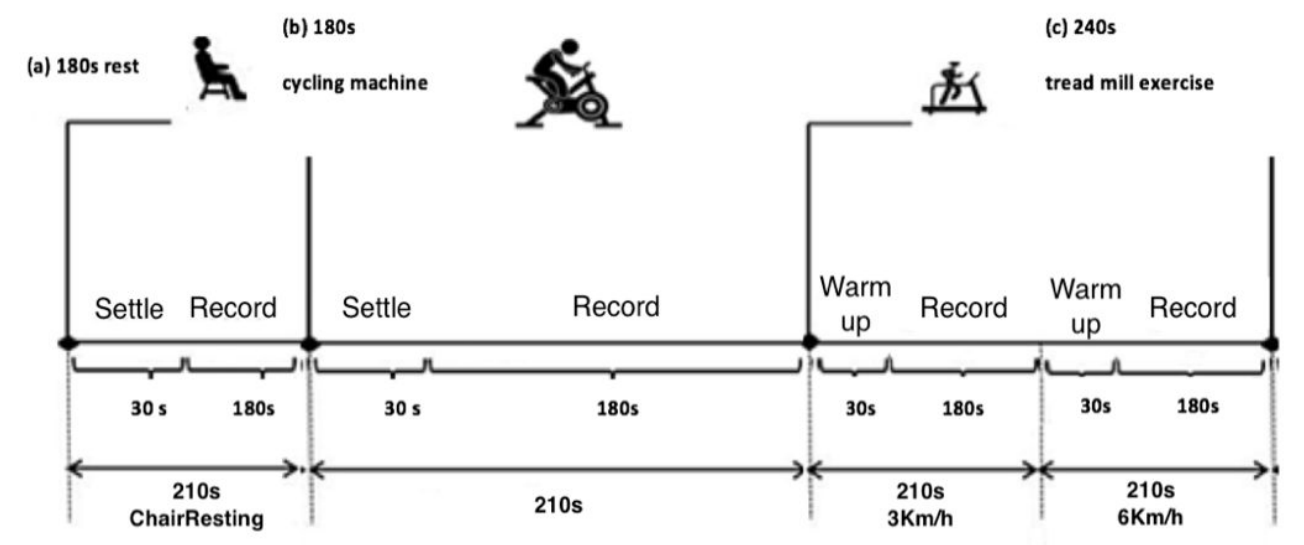

Figure 3.1.1 Diagram of Physiological monitoring protocol

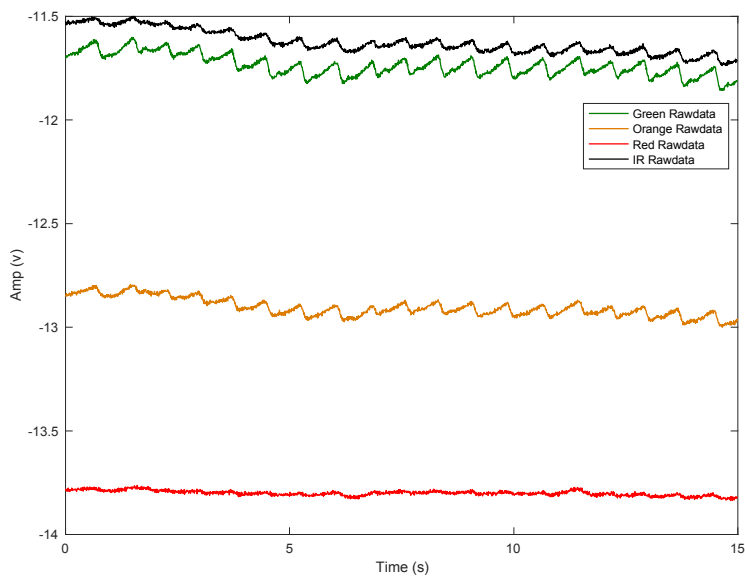

(a) raw data

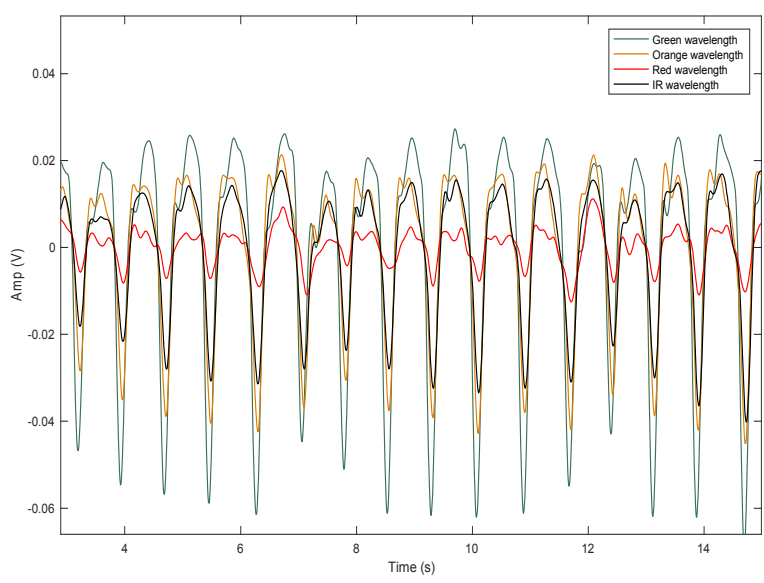

(b) AC components

Figure 3.1.2 Data obtained from OEPS

\subsection{Signal processing for Heart Rate (HR)}

The HR measurement uses the signal obtained at a single wavelength of PPG illumination in order to determine the systolic and diastolic phase of the heart. In particular, a measurement of the separation in time between successive peaks or successive can be used, as shown in figure 3.2.1. 


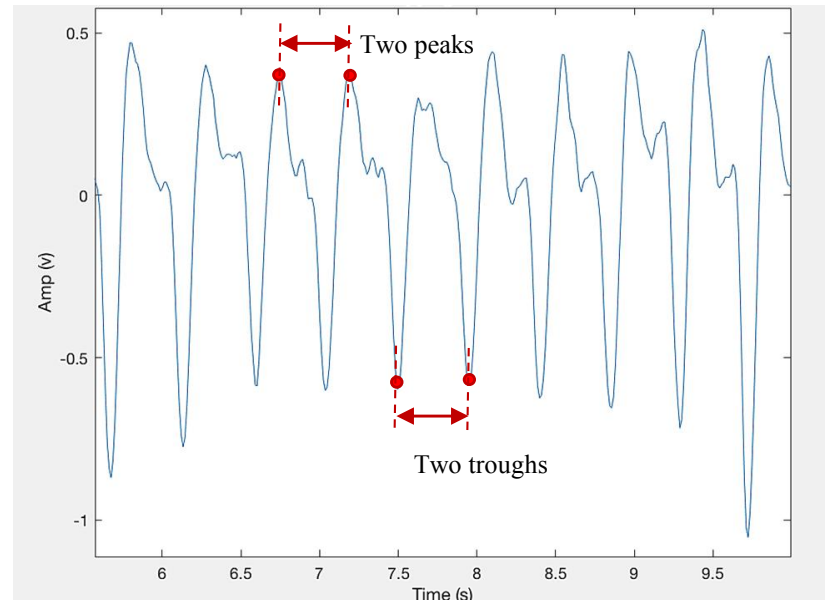

Figure 3.2.1 The PPG signal showing measurements obtained from successive peaks or troughs

Assuming that times of the first and second peaks are $t_{1}$ and $t_{2}$ respectively, then the heart rate is given by

$$
\operatorname{HR}(\mathrm{bpm})=\frac{60}{t_{2}-t_{1}}
$$

\subsection{Signal processing for Oxygen Saturation (SpO2\%)}

The oxygen saturation of the blood is the degree to which oxygen is chemically combined with haemoglobin. $\mathrm{SpO}_{2} \%$ can be determined as the fractional ratio between the concentration of oxygenated haemoglobin and the total haemoglobin present in the blood, as shown in the following equation

$$
\mathrm{SpO}_{2}(\%)=\frac{\left[\mathrm{HbO}_{2}\right]}{\left[\mathrm{HbO}_{2}\right]+[\mathrm{Hb}]} \times 100 \%
$$

where $[\mathrm{Hb}]$ is the concentration of the deoxyhaemoglobin form and $\left[\mathrm{HbO}_{2}\right]$ is the concentration of the oxy-haemoglobin form.

In general, $\mathrm{SpO}_{2} \%$ can be estimated using a PPG from measurements obtained from illuminating the vascular bed with light of two separate wavelengths. In the current work, green and orange light sources are used and for both signals measurements are obtained of the DC and AC components. The DC components can be determined from the raw data as shown in Figure 3.3.1(a) and the AC components can be obtained by determining the positions of peaks and troughs as shown in Figure 3.3.1(b). 


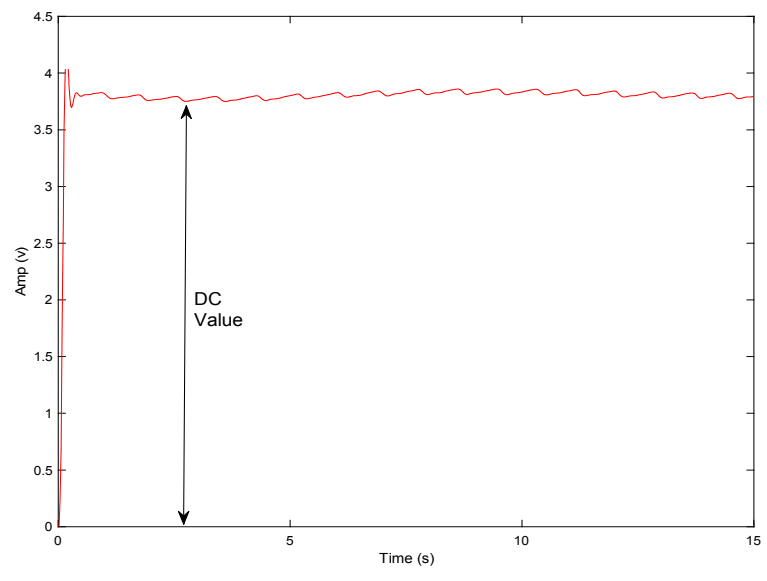

(a) determination of the DC component

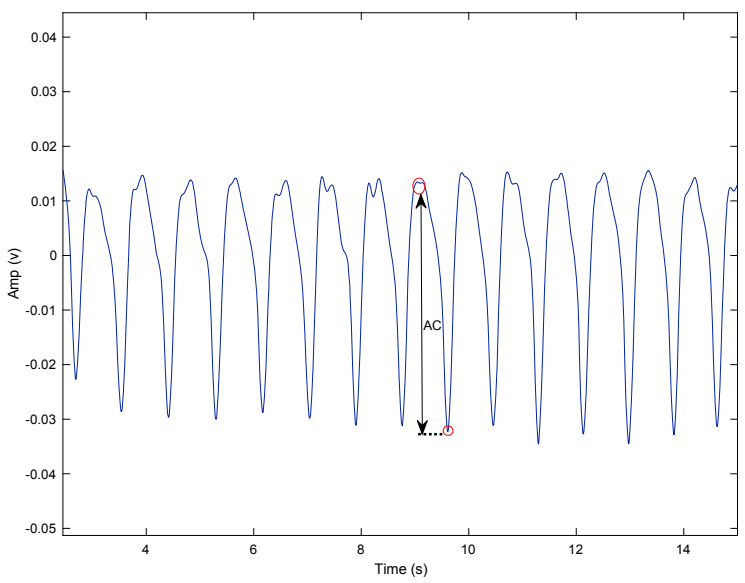

(b) determination of AC amplitude

Figure 3.3.1 Measurement of PPG signals to extract the DC and AC components

A value known as the ratio-of-ratios $(R)$ is then calculated using the following equation.

$$
R=\frac{\left(\frac{A C_{\text {green }}}{D C_{\text {green }}}\right)}{\left(\frac{A C_{\text {orange }}}{D C_{\text {orange }}}\right)}
$$

The oxygen saturation can then be calculated as

$$
\mathrm{SpO}_{2}(\%)=A-B R
$$

where $A$ and $B$ are parameters that can be estimated from a series of readings.

\subsection{Hardware Implementation}

A multi-wavelength opto-electronic patch sensor (OEPS) has been developed to provide to capture PPG signals at green, orange, red and IR wavelengths. A microcontroller is used to control the illumination provided by the PPG sensor and the capture of the reflected signals, as shown in Figure 3.4.1 The lighting is synchronized for the sixteen LEDs, arranged as four sets of LEDs, with the LEDs in each set providing light of the same wavelength. The LED sets are driven in a cyclical sequence (each set of four LEDs are illuminated at the same time, with the LEDs of other of the other sets turned off). The reflected signals are pre-amplified and a de-multiplexer used to determine for each captured signal sample the wavelength of light that was used for illumination. The captured signals are then passed individually to the processer for further processing. 


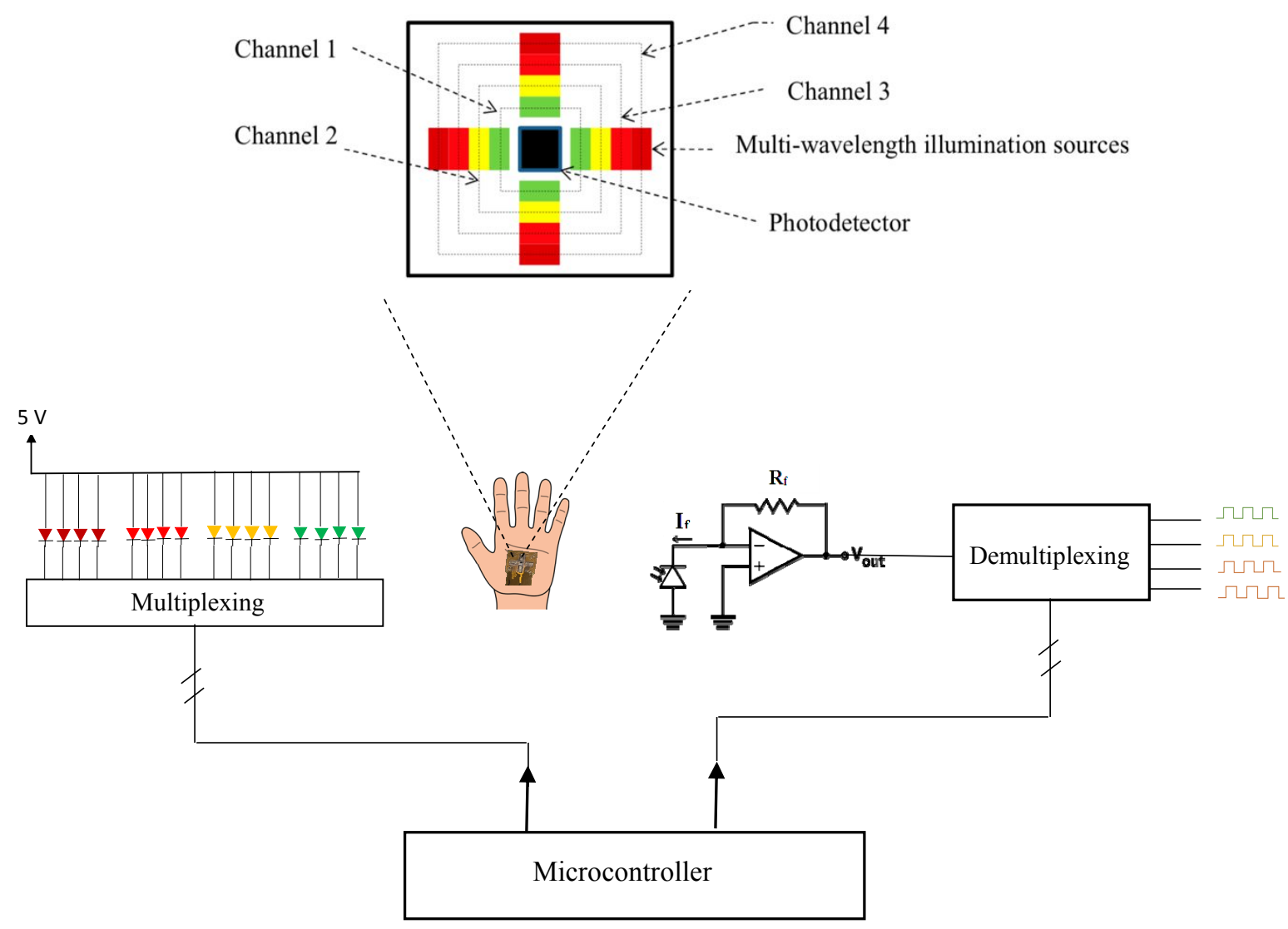

Figure 3.4.1 Microcontroller control of the multiplexing and de-multiplexing operations

OEPS performs the optical capture of pulsatile signals which are then processed electronically using a signal conditioning analogue front-end and embedded microelectronics, before being transmitted using a communications system for storage and off-line analysis. OEPS operates in reflectance mode, giving greater flexibility in the physical sites that can be used for capture, such as the forehead, palm, earlobe or wrist. All data sets obtained from OEPS were collected by the means of a 4-channel PPG board (DISCO4, Dialog Devices Ltd., Reading, Berkshire, UK). The analogue-to-digital conversion used to capture PPG signals was carried out by a 14-bit data acquisition board (DAQ, USB-6009, National Instruments Co., Novato, CA, USA) and the control software of the PPG board was implemented in a LabVIEW GUI (National Instruments Co., USA). A filter with a pass band range of $0.1 \mathrm{~Hz}$ to $10 \mathrm{~Hz}$ was implemented to reduce the influence of extraneous noise and a sampling frequency of $256 \mathrm{~Hz}$ was sufficient to reconstruct the PPG signals according to the Nyquist criteria. The PPG data were displayed in real time to confirm satisfactory signals were being obtained and the captured signals were stored for off-line analysis.

\section{RESULTS}

This section describes the $\mathrm{HR}$ and $\mathrm{SpO}_{2} \%$ results obtained using OEPS and presents a comparison with the measurements obtained using commercial devices. 


\subsection{Heart Rate (HR)}

To compare the performance of OEPS with commercial devices, unpaired $t$-tests and one-way analysis of variance and correlation were used. The agreement between the OEPS and the ECG recorder was evaluated using Bland Altman's 95\% limits of agreement approach. The HR values obtained at each wavelength offered by OEPS and the golden standard obtained from the ECG recorder are shown in Figure 4.1.1 The results show a strong correlation beteen ECG and all the wavelengths provided by OEP

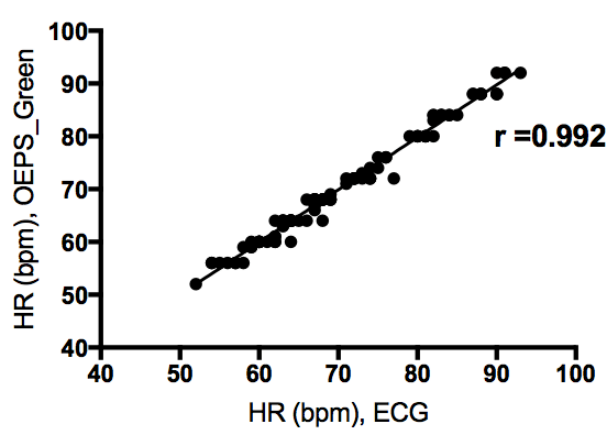

(a) green

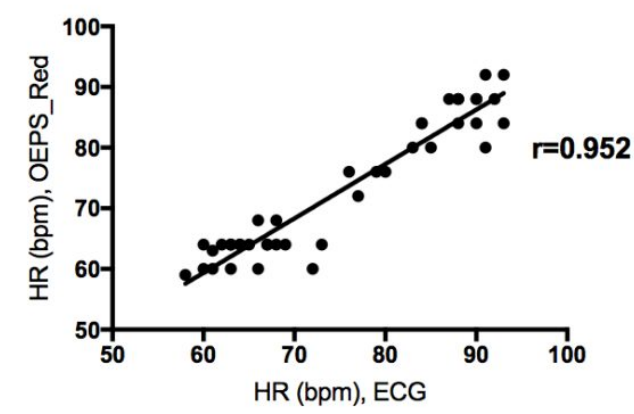

(c) red

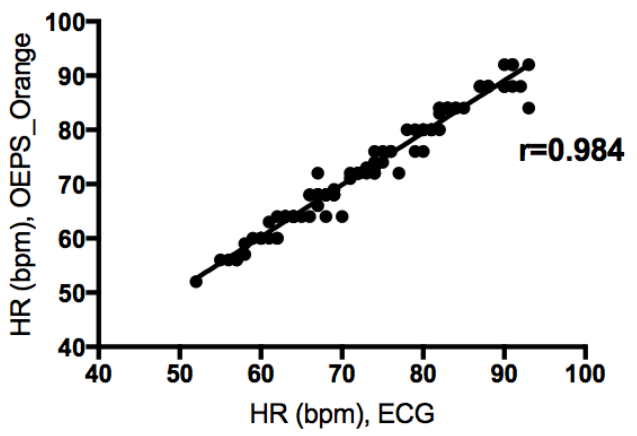

(b) orange

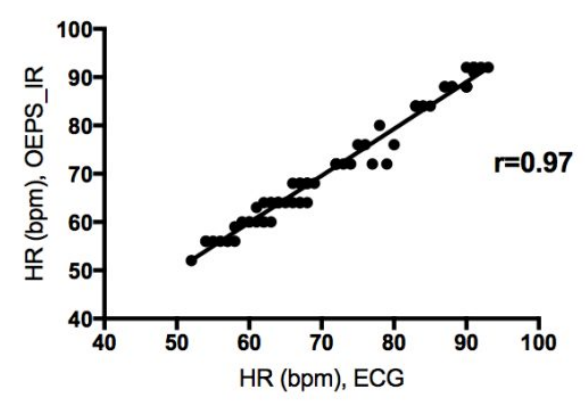

(d) IR

Figure 4.1.1 HR results obtained by the ECG recorder in correlation with OEPS operating at different colors of illumination

In addition, the relationships of the HR readings obtained from the ECG recorder and each wavelength available in OEPS were implemented by using the Bland-Altman and the results can be seen in Figure 4.1.2. 


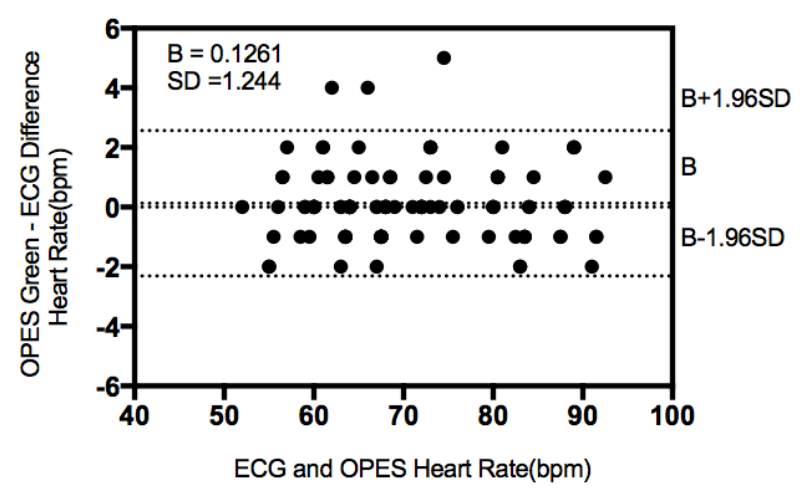

(a) green

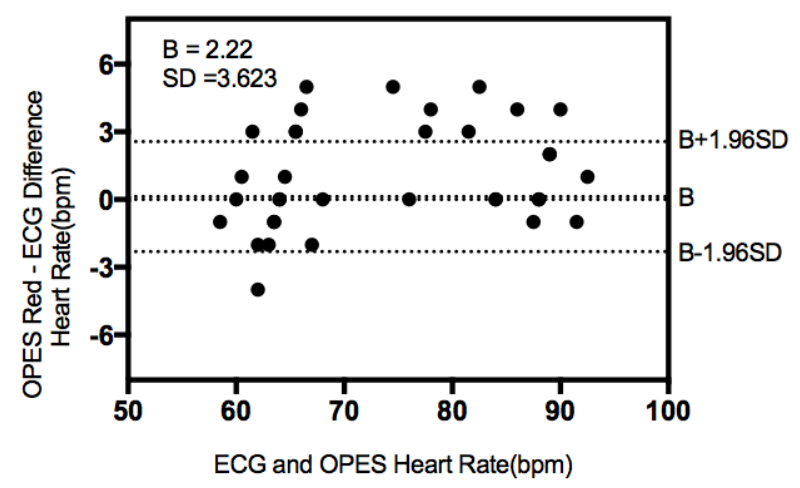

(c) red

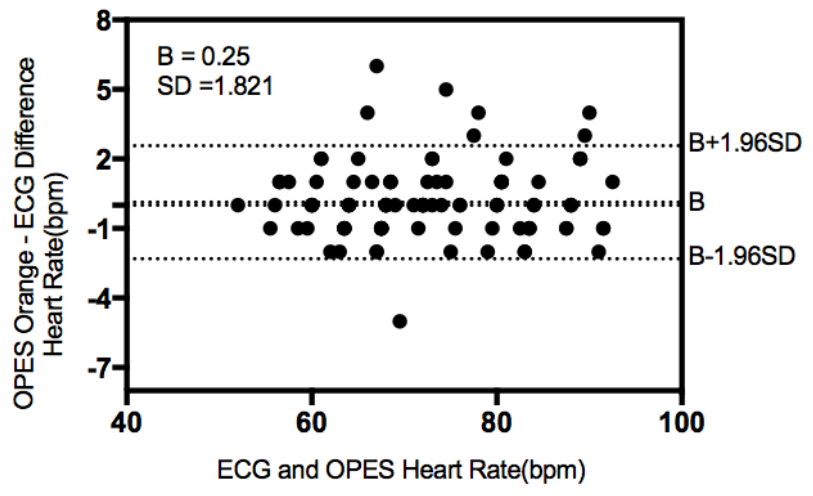

(b) orange

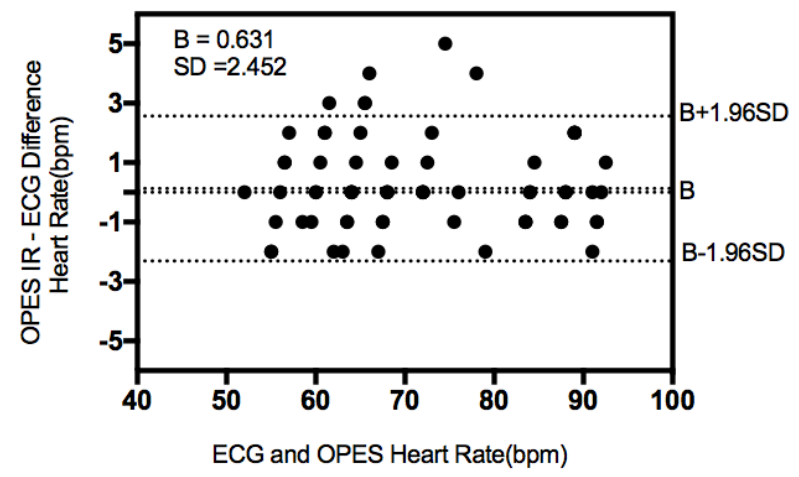

(d) IR

Figure 4.1.2 Bland-Altman plot showing differences in HR between the ECG recorder and OEPS operating at different colors of illumination

Table 1 shows the result of comparisons performed for a number of the commercial devices and OEPS using the $t$ - test. The table shows mean values obtained for the ECG recorder as well as the mean values obtained from the compared device. The mean difference shows only small variations when the OEPS wavelength is changed, with the probability value consistently being 0.999 . The HRM-3200 device produced values that were not in close accordance with the other systems used and the cause of this has yet to be determined. 
Table 1 HR readings from the ECG recorder compared with both commercial devices and OEPS

\begin{tabular}{|c|c|c|c|c|c|}
\hline \multirow{2}{*}{ Comparison test } & \multicolumn{3}{|c|}{ mean } & \multirow{2}{*}{ p value } & \multirow{2}{*}{ significant? $p<0.05$} \\
\hline & ECG & second device & difference & & \\
\hline ECG \& Honour S1 & 73.15 & 73.73 & -0.5833 & 0.999 & No \\
\hline ECG \& MZ-3 & 73.15 & 74.65 & -1.5 & 0.985 & No \\
\hline ECG \& HRM-3200 & 73.15 & 76.93 & -3.783 & 0.446 & No \\
\hline ECG \& Green & 73.15 & 73.86 & -0.7144 & 0.999 & No \\
\hline ECG \& Orange & 73.15 & 73.86 & -0.7144 & 0.999 & No \\
\hline ECG \& Red & 73.15 & 73.61 & -0.4598 & 0.999 & No \\
\hline ECG \& IR & 73.15 & 73.78 & -0.6297 & 0.999 & No \\
\hline
\end{tabular}

Figure 4.1.3 shows the mean and standard error of difference (SED) of all devices The HRM-3200 and MZ-3 are in significant disagreement with ECG reference value.

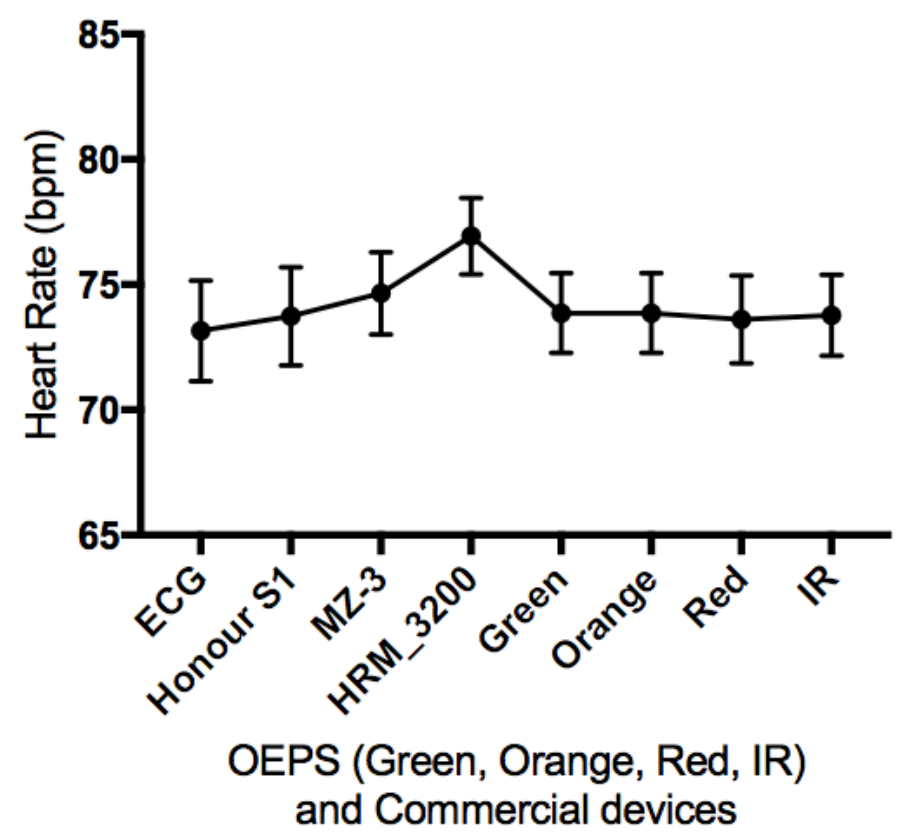

Figure 4.1.3 Mean and Standard Error Difference of the commercial devices and OEPS

\subsection{Oxygen Saturation $\left(\mathrm{SpO}_{2} \%\right)$}

The green and orange OEPS wavelengths were used to estimate $\mathrm{SpO} \%$ and the results were compared with those of a commercial pulse oximetry device $\left(\mathrm{TempIR}{ }^{\mathrm{TM}}\right)$. The results show good agreement and a correlation of $\mathrm{r}=0.98$, as shown in Figure 4.2.1. 


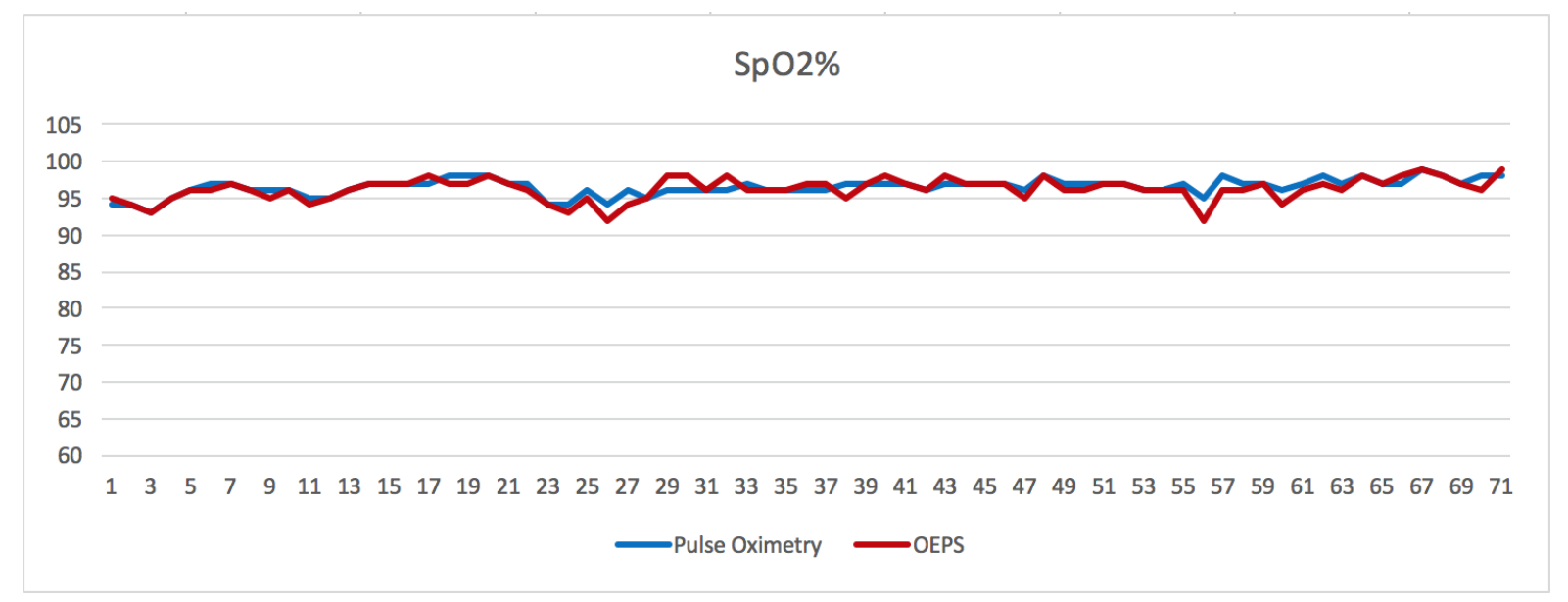

Figure 4.2.1 $\mathrm{SpO}_{2} \%$ data obtained from OEPS and a commercial pulse oximetry device.

An unpaired $t$-test was carried out on the $\mathrm{SpO}_{2} \%$ data and a strong relationship was found between the $\mathrm{SpO}_{2} \%$ measured by using the OEPS and using pulse oximetry as well as vice versa $(\mathrm{r}=0.982)$. No significant difference was found $(\mathrm{p}=0.894)$, as shown in Figure 4.2.2.

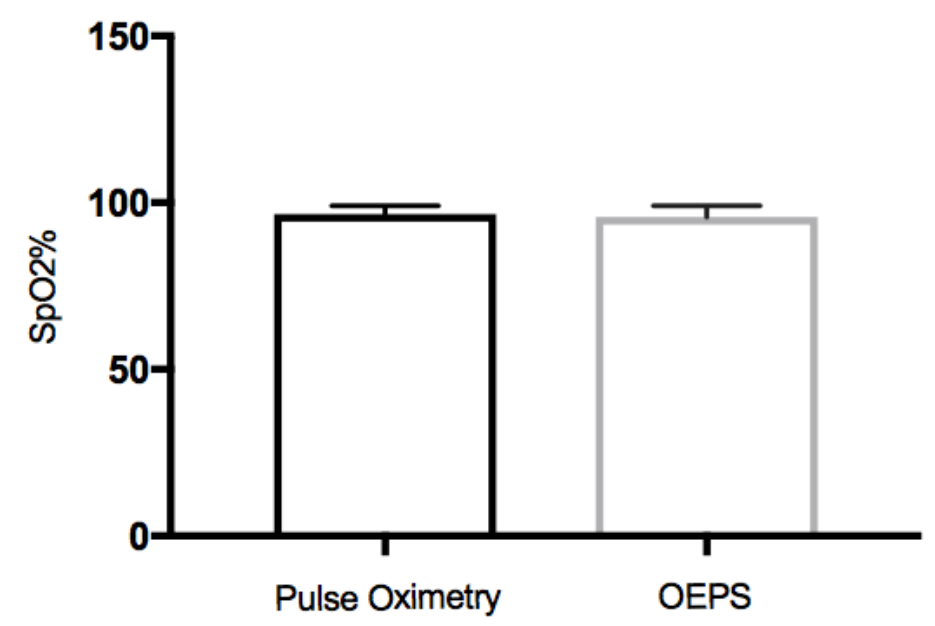

Figure 4.2.2 Mean and standard error mean of for the pulse oximetry device and OEPS 


\section{DISCUSSION}

The OEPS sensor provides multi-wavelength illumination that is able to improve the performance of opto-physiological monitoring. OEPS is designed to have four LEDs to provide illumination at each wavelength, generating a more even illumination of the skin. This results in OEPS being less affected by small movements, for even if the incident light from one LED, or even one set of LEDs, becomes misaligned with the arteries, at least one of the other source lights in OEPS is still likely to have sufficient alignment with the area of interest to be able to capture a useful reflected signal.

OEPS provides the opportunity to capture PPG signals more reliably by providing the opportunity to select an appropriate wavelength or set of wavelengths according to the skin type and skin thickness of individual subjects. It was found during the study that the signals obtained from green and orange illumination were more robust when a subject was carrying out physical activities, probably due to the fact that these signals give little penetration beneath the surface of the skin. Consequently, green and orange lighting are wavelengths that are likely to be better suited to obtaining measurements during normal daily activities.

Moreover, the green and orange wavelength have narrow band wavelength and a peak of $525 \mathrm{~nm}$ (green), 590nm(orange) which assist to focus, absorbed effectively and maximize the SNR of pulsatile dynamic PPG. Furthermore, they could be used for new-born baby and child-care as their skin are too thin. Both green and orange wavelength facilitate stable and accurate reading of $\mathrm{HR}$ and $\mathrm{SpO}_{2} \%$.

Red and IR wavelengths are used in most commercial and research devices for the estimation of $\mathrm{SpO}_{2} \%$. As can be seen in the raw data collected for Figure 3.1.2, the amplitude of red signal was consistently less than that of the other wavelengths, thus making its SNR lower, and meaning it is likely to be corrupted as a result of movement. Referring to Figure 2.3, it can be seen that there are differences of in the absorption of $\mathrm{Hb}$ and $\mathrm{HbO}_{2}$ at the wavelengths identified as green and orange, making these better suited for the extraction of $\mathrm{SpO}_{2} \%$ values. At these wavelengths the penetration into the skin is significantly shallower compared with red and IR, implying that the use of green and orange for $\mathrm{SpO}_{2} \%$ value estimation likely to be more reliable during physical activity.

As depicted in the Bland-Altman plot in Figure 4.1.2, the HR data is most likely to be in the acceptable range of B \pm 1.96 SD of the difference between the ECG and the OEPS signals. The bias in the HR measured using OEPS and ECG was found to be low and the limits of agreement were acceptable. However, the bias $(B=2.22)$ of the red data are not compatible but not satisfactory as well. The one-way ANOVA analysis showed that there is no significant difference between OEPS and ECG. Referring to Table 1, the mean of difference of the red wavelength (0.4598) is less than that of green (0.7144), orange (0.7144) and IR (0.6297), however this results from corrupted data being largely omitted and so leaving fewer data values for the calculation of the mean of difference. The oxygen saturation that extracted from the OEPS is comparable with pulse oximetry reading with correlation of $r=0.98$ and $p=0.894$ as shown in Figures 4.2.1 and 4.2.2.

\section{CONCLUSION}

It has been demonstrated that a multi-wavelength opto-electronic patch sensor (OEPS) is effective at detecting the physiological parameters $\mathrm{HR}$ and $\mathrm{SpO}_{2} \%$ in a range of application scenarios. A comparison involving the measurement of HR showed that OEPS was able to provide readings in good agreement with commercial ECG devices. Four wavelengths of illumination were used to extract HR, but the results show that green illumination has the greatest modulation among the wavelengths used in the study, regardless of skin type. Thus, the HR signals were mainly obtained by using green wavelength illumination and $\mathrm{SpO}_{2} \%$ by using green and orange (which also generated a strongly reflected signal).

Due to the multi-wavelength nature of OEPS, illumination adaption can be performed in OEPS to measure HR with a certain degree of tolerance to different skin types and skin thickness. OEPS would appear to be of particular value in sport physiological monitoring applications, but is also likely to have applications in adult and child healthcare, where it can provide cost-effective monitoring and assessment. Furthermore, OEPS could be consolidated with already-available wearable and smart devices for real-time monitoring and assessment. 
Several challenges remain. Appropriate siting is of great importance in improving the robustness of PPG devices, specifically in determining the location that provides the best access to a rich source of peripheral blood vessels, perhaps the back or chest. Further studies will investigate the effects of motion artefacts and how they can be reduced using additional sensing and electronics. One observation from the current study is that OEPS will be able to provide an optophysiological monitoring approach that will be well suited to incorporation into wearable devices. As part of the development of a wearable solution, the simplification of the controlling electronics and the embedding of the data processing algorithms will be considered. For OEPS this will be achieved through improvements in packaging, automated adaption to different subjects and as well as providing users with recommendations for suitable operational procedures for different application areas.

\section{ACKNOWLEDGEMENTS}

The authors would like to acknowledge Loughborough University and the Ministry of Higher Education in the Kingdom of Saudi Arabia for supporting this research. We also would like to convey our special thanks to Mortara Instrument UK Ltd for letting us a trial ECG device.

\section{REFERENCES}

[1] Lopez, S., et al, "Pulse Oximeter Fundamentals and Design," Free- scale Semiconductor Inc. application note document No AN4327 Rev 1- 09, 4327, (2011).

[2] Toshiyo, T., Yuka, M., Masaki, S., Masaki, Y., "Wearable Photoplethysmographic Sensors-Past and Present," J Electronics, 3, 282-302, (2014).

[3] Jayasree V.K, "Selected cardiovascular studies based on photoplethysmography technique," International School of Photonics Cochin University of Science and Technology Cochin - 682 022, India, (2009).

[4] H3 SYSTEM., "HRM-3200 Heart Rate Monitor" https://usermanual.wiki/H3-SYSTEM/HRM-3200, (Nov 22th, 2017).

[5] Huawei., "Honor Watch S1," http://www.smartwatchspecifications.com/Device/huawei-honor-watch-s1-glorywatch-s1/,(Dec 5th 2017).

[6] MZ-3Belt., "My Zone Belt," http://myzone.org/meet-the-mz3/,(Dec 12th 2017).

[7] Nonin Medical Inc., "Nonin WristOx 3100," Medical Product Service GmbH Nonin, (2006).

[8] U. Anliker, et al, "AMON: a wearable multiparameter medical monitoring and alert system," IEEE Trans. Inf. Tech. Biomed 8, 415-427 (2004)

[9] Grossman, P., "The Life Shirt: a multi-function ambulatory system monitoring health, disease, and medical intervention in the real world," Stud. Health Technol. Inform, 108, 133-141(2004).

[10] Park, S., Jayaraman, S., "e-Health and quality of life: the role of the Wearable Motherboard," Stud. Health Technol Inform, 108, 239-252 (2004).

[11] Fruin, M. L., Rankin, J. W., "Validity of a multi-sensor armband in estimating rest and exercise energy expenditure," Med Sci Sport Exerc, 36, 1063-1069 (2004).

[12] Pandian, P. S., et al, "Smart Vest: wearable multi-parameter remote physiological monitoring system," Med Eng Phys, 30, 466-477 (2008).

[13] K. Lee, K. Matsumura, P. Yamakoshi et al, "Comparison between Red, Green and Blue Light Reflection Photoplethysmography for Heart Rate Monitoring During Motion". Conf Proc IEEE Eng Med Biol Soc.10, 4577 (2013).

[14] Alzahrani, A.A. "A Reflectance OptoelectronicPatch Sensor for Physiological Monitoring with Motion Artefacts Reduction," Ph.D. Thesis, Loughborough University, Leicestershire, UK 2016. 
[15] Alzahrani. A, Hu. S, et al, "A Comparative Study of Physiological Monitoring with a Wearable OptoelectronicPatch Sensor (OEPS) for Motion Reduction". Biosensors, 8, 5 (2), 288-307 (2015).

[16] Yan. L, Hu. S, et al, "A Multi-Wavelength Optoelectronic Patch Sensor to Effectively Detect Physiological Changes against Human Skin Type," Biosensors, 7, 22 (2017).

[17] Strojnik, M. Pae, G., "Spectral dependence of absorption sensitivity on concentration of oxygenated hemoglobin: pulse oximetry implications," J Biomed Opt 18(10),108001 (2013).

[18] Taxis Instrument.,"Miniaturized Pulse Oximeter Reference Design," TIDA-003, (2014).

[19] Uyuklu, M., Canpola,t M., et al., "Wavelength selection in measuring red blood cell aggregation based on light transmittance," J Biomed Opt, 16(11), 117006 (2011).

[20] Nitzan, M., De Boer, H., Turivnenko, S., et al., "Power spectrum analysis of the spontaneous fluctuations in the photoplethysmographic signal." J of Basic and Clinical Physiology and Pharmacology, 5 , 269-276 (1994).

[21] Sangeeta B., Laxmi S. A., "Real time analysis of PPG signal for measurement of SpO2 and pulse rate," Intr. J. Comput App, 36 (11), (2011).

[22] Omar A., Armin B., "Adaptive filtering by non-invasive vital signal monitoring and diseases diagnosis," SBN 978953-307-306-4, CC BY-NC-SA 3.0 license (July 5, 2011) under. () The Author(s).

[23] Sonoda, K., et al, "Wearable Photoplethysmographic Sensor System with PSoC Microcontroller," Fifth Int'l. Conf. Emerg. Trends Eng. Tech. (ICETET), (2012).

[24] Vizbara, V., Sološenko, A., Stankevičius, D., Marozas. V., "Comparison of green, blue and infrared light in wrist and forehead photoplethysmography," Conf. Biomed. Eng., Biomedical Engineering Institute, Kaunas University of Technology, Lithuania (2011).

[25] Fodor, L., Ullman, Y., Elman M., "Aesthetic Applications of Intense Pulsed Light," London: Springer London, P. 133 (2011) 